\title{
Cáncer de mama en mujeres pre y perimenopáusicas
}

Breast cancer in pre and perimenopausal women

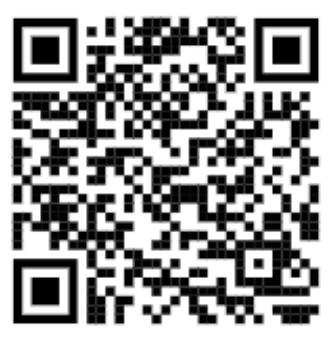

${ }^{1}$ Médico general, graduada de la Universidad de Ciencias Médicas (UCIMED).

Código médico 15306

catacoto1493@hotmail.com

${ }^{2}$ Médico general, graduado de la Universidad de Ciencias Médicas (UCIMED).

Código médico 15663

mjimenezvi18@gmail.com

${ }^{3}$ Médico general, graduada de la Universidad Latina de Costa Rica (U.Latina).

Código médico 15569

dra.snaranjo@gmail.com

\begin{abstract}
${ }^{1}$ Dra. Catalina Coto Chaves Instituto de psicopedagogía integral colegio Issac Martin (IPICIM), San José, Costa Rica catacoto1493@hotmail.com https://orcid.org/0000-0001-6152-0235

${ }^{2}$ Dr. Mauricio Jiménez Víquez Solumed- Hospital La California, San José, Costa Rica mjimenezvi18@gmail.com

https://orcid.org/0000-0003-4392-5591

${ }^{3}$ Dra. Stephanie Naranjo Alfaro Investigadora independiente, San José, Costa Rica dra.snaranjo@gmail.com

https://orcid.org/0000-0001-9293-0616

$\begin{array}{lcc}\text { RECIBIDO } & \text { CORREGIDO } & \text { ACEPTADO } \\ 28 / 02 / 2019 & 04 / 03 / 2019 & 16 / 04 / 2019\end{array}$
\end{abstract}

\section{RESUMEN}

El cáncer de mama es una patología que, aunque tiene mayor incidencia en mujeres mayores, se ha visto en incremento en féminas pre y perimenopáusicas. Siendo el cáncer de mayor diagnóstico y muerte en mujeres. Su etiología no es completamente conocida, no obstante, en este artículo revisamos los factores de riesgo más importantes, así como la importancia del examen físico, imágenes complementarias y biopsia para la detección y diagnóstico. También los diferentes tratamientos que se individualizan de acuerdo al paciente.

PALABRAS CLAVE: neoplasias de la mama; detección precoz del cáncer; mamografía; ganglio linfático centinela; inmunohistoquímica; tamoxifeno.

\section{ABSTRACT}

Although breast cancer is a pathology that has more incidence in older women, there has been an increment in pre and perimenopausal females. Being the most diagnosed cancer among women and the one who has more deaths. The etiology isn't completely known, however in this article we reviewed the most important risk factors, as well as the 
importance of physical exam, complementary images and biopsy for detection and diagnosis. Also the different treatments, individualized according to the patient.

KEYWORDS: breast neoplasms; early detection of cancer; mammography; sentinel lymph node; immunohistochemistry; tamoxifen.

\section{INTRODUCCIÓN}

La transición perimenopausia / menopausia se define por cambios hormonales y del ciclo menstrual que suceden unos cuantos años antes y 12 meses después del último periodo menstrual resultante de una menopausia natural. El cáncer de mama es el tumor más frecuente en mujeres. Es la proliferación maligna de células epiteliales que revisten los conductos o lobulillos de tal glándula, abarca un espectro de trastornos benignos y malignos que se manifiestan muy a menudo con dolor mamario, secreción por el pezón o una masa palpable (1). Los trastornos benignos predominan en las premenopáusicas jóvenes, mientras que las tasas de malignidad aumentan con la edad. Para valorar los trastornos mamarios casi siempre es indispensable la combinación de una anamnesis cuidadosa, exploración física, estudios de imagen y, cuando está indicada, biopsia (1). Este artículo pretende la revisión de la patología en mujeres pre y perimenopáusicas, sus manifestaciones, factores de riesgo y abordaje.

\section{HISTORIA}

El primer manuscrito que documenta sobre el cáncer de mama es el papiro de Edwin Smith, escrito aproximadamente en el año 1600 a.c. En él se describen 8 casos de tumores o úlceras que fueron tratados con cauterización, con una herramienta llamada "la orquilla de fuego", o con extirpación $(1,2)$. Asimismo, el primer reporte de cáncer de mama data del año 3000 a 2500 a.c, y fue diagnosticado en un hombre (1).

En los siglos XVI y XVII, los cirujanos Andrea Vesalio, Ambrose Paré, Cabral, Miguel de Servet y otros, aumentaron el conocimiento sobre la enfermedad, dando a conocer su difusión vía linfática a los ganglios axilares, y como ésto era signo de mal pronóstico (2). Más adelante, también en el siglo XVII, se da la asociación entre el cáncer de mama y los nódulos linfáticos axilares. Siendo Jean Louis Petit (1674-1750), cirujano francés y luego Benjamín Bell (1749-1806), los primeros en realizar la mastectomía radical (remoción de nódulos linfáticos, tejido mamario y músculos pectorales con conservación de la piel y el pezón si estos no estaban afectados) (2).

Charles Moore enunció principios de abordaje quirúrgico en el que establecía que el tumor no debía ser visto ni dividido en la operación, y que las recurrencias del cáncer de mama provienen de la dispersión del cáncer primario en forma centrífuga y no por otra causa orgánica. Por lo que concluyó que se debía extirpar completamente del órgano, cuidando de implicar el borde esternal y cuidando de 
extraer, junto con la masa, la piel y tejidos circundantes afectados por continuidad (2).

Seguidamente Stewart Halsted, modificó la mastectomía, creando la mastectomía radical de Halsted. En la que se extirpa la glándula, la piel que la cubre, la fascia y músculo pectoral, y el paquete ganglionar axilar (1,2). Posteriormente varios cirujanos experimentaron nuevas técnicas basadas en experiencias personales, dando lugar a la Mastectomía modificada de Patey, la Mastectomía supraradical de Dahl-Ivern, la Mastectomía simple y la Mastectomía subcutánea (1).

Tras la invención de los rayos $\mathrm{X}$ y el aporte del matrimonio Curie, se inició el uso de radioterapia. Luego, en el siglo XIX se incorporó el uso de compuestos arsenicales y en periodos más actuales el uso de mostazas nitrogenadas, ambos como quimioterapéuticos (2).

\section{EPIDEMIOLOGÍA}

Según las estadísticas de 2018 de la American Cancer Society se encuentran 266,000 casos de cáncer de mama por año, y es responsable de al menos 40,000 muertes (3). Las estadísticas mundiales informan que es el cáncer de mayor frecuencia diagnóstica y de muerte en las mujeres, reportando un $14 \%$ de las muertes de un total de $23 \%$ de muertes por cáncer. Siendo la causa líder en mujeres de países económicamente desarrollados (4). Aun así, los índices documentan una disminución desde 1997 a 2007 de $1.8 \%$ por año y de $1.9 \%$ de 2008 a 2012 (3,4). Disminuyendo mayormente en mujeres caucásicas que en afroamericanas (4).En México y Latinoamérica el cáncer de mama en mujeres menores de 40 años, por el contrario, va en aumento, reportándose un $11 \%$ frente al $3 \%$ de países desarrollados como EUA, Canadá y Europa. En mujeres menores de 40 años está asociado a un $15 \%$ de probabilidad a factor genético secundario (5). Por lo general suele ser más agresivo en pacientes jóvenes y mayormente son carcinomas inflamatorios, donde normalmente no se presentan masas y se manifiesta con síntomas de mastitis (1).

Se ha asociado a los cambios de incidencia, el impacto de la industrialización de la sociedad (cambios en el consumo de grasas, peso corporal, menarca $y / 0$ lactancia materna, $y$ patrones reproductivos como la disminución de los embarazos y edad mayor al primer parto), además de la discontinuidad de la Terapia de reemplazo hormonal y el aumento en el tamizaje mediante mamografía $(5,6)$.

\section{FISIOPATOLOGÍA}

Hasta el momento, su etiología en gran parte es desconocida, pero se conoce que en esta patología los factores de riesgo suelen ser de suma importancia. Los antecedentes familiares, juegan un papel fundamental, así también los genes de alta susceptibilidad al cáncer como BRCA1, BRCA2 y TP53 o algunos polimorfismos de un solo nucleótido en FGFR2, TNRC9, MAP3K1, LSP1, CASP8 y TGFB1 (7). Se conocen mutaciones genéticas somáticas por subtipo molecular intrínseco, las cuales se dividen en los tumores luminales $A$ y $B$, estas tienen perfiles mutacionales cuyas mutaciones se encuentran en los reguladores de la diferenciación luminal 
en la glándula mamaria normal, PI3K, MAP3K1, GATA3, FOXA1, PI3K, MAP3K1, GATA3, FOXA1, TBX3 y los tumores de tipo basal, los cuales por lo general tienden a tener un mayor número de mutaciones somáticas e inestabilidad genómica que los tumores luminales $A$ y $B$, pero frecuentemente tienen un número menor de genes mutados (7).

En cuanto a su patogenia, se han encontrado dos posibles modelos sobre el origen de los subtipos de cáncer de mama, el modelo de origen celular se conoce que cada subtipo de tumor se origina de diferentes células madre 0 progenitoras y el modelo de evento específico, en el cual la célula que da origen puede ser la misma para diferentes subtipos, con diferencias en el fenotipo tumoral determinadas por eventos genéticos y epigenéticos adquiridos (8).

Se cree que la progresión se produce a través de etapas patológicas y clínicas definidas.

- La progresión puede ser impulsada por la acumulación de cambios genéticos combinados con la expansión y selección clonal (8).

- Interacciones entre el epiteliomesenquimal, las células del microentorno y las moléculas de la matriz extracelular las cuales han demostrado ser importantes tanto para el desarrollo normal como para la tumorigénesis de la mama (7).

- La señalización autocrina / paracrina anormal también desempeña un papel en la progresión del tumor de mama; Las quimiocinas reguladas al alza en las células mioepiteliales tumorales y los miofibroblastos aumentan la proliferación, migración e invasión de las células tumorales y promueven la angiogénesis y la diseminación metastásica (7).

- Las mujeres afroamericanas y las mujeres en África desarrollan tumores de tipo basal (con mayor riesgo de metástasis) con más frecuencia que las mujeres de ascendencia europea, lo que sugiere que un microambiente asociado con genotipos específicos influye en el riesgo de metástasis (8).

\section{FACTORES DE RIESGO}

Historia familiar: constituye uno de los factores de mayor riesgo, especialmente si se desarrolló en la premenopausia, si fue bilateral o sucedió en familiares de primer grado, con un incremento del riesgo a mayor número de familiares afectados, ya sea por vía materna o paterna (9). Existen algunos tipos de cáncer relacionados con la historia familiar, uno es el descrito como cáncer familiar y otro es el que podríamos llamar cáncer hereditario (10). En el primer caso, existen varios miembros de la misma familia afectados por la enfermedad, sin que exista transmisión autosómica dominante, que podría estar modulada por factores ambientales y de estilo de vida. El cáncer de mama hereditario se produce como resultado de una transmisión autosómica dominante, afectando a múltiples generaciones, estos suponen tan sólo un $5 \%$ de todas las neoplasias de mama, los familiares un $10 \%$, y el resto serían cánceres esporádicos (10).

Alteraciones genéticas: hasta un 10\% de todos los cánceres ocurren por defectos genéticos hereditarios y gracias a los avances en la biología molecular, se 
han descubierto genes cuyas mutaciones predisponen a algunos tipos de neoplasias. Ejemplos de ellos son el BRCA-1 y BRCA-2, que aumentan el riesgo de desarrollar cáncer de mama, ovario, tubas uterinas, páncreas, vía biliar, vesícula biliar, carcinoma gástrico y melanoma. De los carcinomas mamarios un $5-10 \%$ son hereditarios de una forma autosómica dominante y $25 \%$, asocia mutación en los genes BRCA (11). La mutación BRCA-1, se da principalmente en pacientes jóvenes premenopáusicas con antecedentes familiares positivos, es más frecuente en judíos y de tendencia bilateral. Conlleva una probabilidad de 56$90 \%$ de desarrollar Cáncer de mama y 40 $50 \%$ de neoplasias ováricas (11). Con respecto al BRCA-2, el riesgo es algo menor, con probabilidades de $37-84 \%$ de desarrollar cáncer de mama y el grupo etáreo es un poco más amplio, en mujeres no tan jóvenes (11).

Edad: el riesgo aumenta con la edad, solo el $5 \%$ de estos tumores ocurren en mujeres menores de 40 años (10). Aunque el cáncer de mama se diagnostica a cualquier edad, la incidencia antes de los 30 años es baja, la frecuencia aumenta progresivamente hasta alcanzar una meseta entre los 45 y los 55 años. La mayor dificultad diagnóstica a edades tempranas es por la menor sensibilidad de la mamografía en mamas jóvenes, más densas, y la mayor proporción de cáncer avanzado a estas edades (10).

Factores dietéticos y estilo de vida: la vida sedentaria asociada a la obesidad sería otro de los parámetros que aportarían mayor riesgo (12). Se ha demostrado que existe una correlación entre obesidad central y cáncer de mama en mujeres posmenopáusicas debido a que en las mujeres obesas aumenta la tasa de estrógenos circulantes procedentes del tejido graso (9). Estas mujeres tienen en sangre valores más altos de estrona y de estradiol que las de peso normal. Paradójicamente, mientras que la obesidad en mujeres menopáusicas es un factor de riesgo, en mujeres jóvenes se ha visto un efecto protector, probablemente a causa de la influencia de los ácidos grasos poliinsaturados que producen hiperinsulinemia, la cual favorece la disfunción ovárica con ciclos anovulatorios con menor tasa de estrógenos circulantes (10).

El consumo moderado de alcohol también aumenta el riesgo, por un mecanismo desconocido, se conoce que el consumo a partir de $10 \mathrm{~g}$ al día podrían incrementan el riesgo, ascendiendo un $7 \%$ el riesgo relativo por cada $10 \mathrm{~g}$ de ingesta al día. La administración de ácido fólico complementario al parecer modifica el riesgo en mujeres alcohólicas. La administración de dosis menores de ácido acetilsalicílico de forma crónica se ha vinculado con una mayor incidencia de cáncer de mama $(9,10)$.

Radiación: la mama es un órgano muy sensible a la radiación por lo cual es un elemento de riesgo mayormente a menor edad (9). Aquellas que han estado expuestas antes de los 30 años a la radiación de la forma de múltiples fluoroscopias o el tratamiento de la enfermedad de Hodgkin muestran un incremento importante en el riesgo de presentar cáncer de mama $(10,12)$. 


\section{FACTORES REPRODUCTIVOS Y HORMONALES}

Ciclos ovulatorios: la menarca a edad temprana (antes de los 12 años) o la menopausia tardía (después de los 55 años), se relaciona con inicio más temprano y prolongado de ciclos ovulatorios y mayor riesgo de cáncer mamario. $(10,12)$ Por el contrario, la menopausia temprana, ya sea natural o quirúrgica, se relaciona con menor riesgo de cáncer de mama (13).

Embarazo: la mama es única entre todos los órganos humanos, existe como un primordio durante la primera década de vida aproximadamente, luego entra a un estado de proliferación intensa en la menarca y no madura completamente hasta el primer parto de un lactante vivo (13). El epitelio mamario inmaduro es más susceptible a los carcinógenos que el epitelio posterior a la lactación, por lo tanto, entre más tardío sea el primer parto, mayor es el riesgo. Con respecto a la paridad, el riesgo de presentar tumores de mama se incrementa en el periodo inmediatamente posterior al nacimiento, el embarazo genera concentraciones muy altas de estradiol circulante que se relacionan con un aumento transitorio del riesgo a corto plazo, no obstante, el embarazo también induce la diferenciación terminal del epitelio mamario y representa un alivio a los ciclos ováricos, por lo que a largo plazo el efecto es protector (12). Cada embarazo a término disminuye un $25 \%$ el riesgo, y las mujeres que han tenido 5 partos a término presentan la mitad del riesgo de cáncer de mama que las mujeres nulíparas. Por consiguiente, el aumento en la paridad se relaciona con un menor riesgo de por vida (9).

Tratamiento de sustitución hormonal: el tratamiento de sustitución hormonal a base de estrógenos conjugados y progestágenos se ha restringido sus indicaciones en la actualidad, por incrementar el riesgo de tumores mamarios y de episodios cardiovasculares adversos, aunque disminuye el riesgo de fracturas de huesos y cáncer colorrectal (9). El riesgo es mayor con el uso prolongado y con el intervalo más corto entre el inicio de la menopausia y el inicio del medicamento; se observó que, si se practicaba durante seis a siete años, se duplicaba el riesgo del cáncer mamario. Los niveles más elevados de estradiol en la posmenopausia incrementan el riesgo, que también parece aumentar con niveles elevados de prolactina y factor 1 de crecimiento tipo insulínico (10). La utilización de dietilestilbestrol durante el embarazo entre los 40 y 60 años incrementó un $30 \%$ el riesgo de desarrollar cáncer de mama (10). En perspectiva, al parecer se produce un número mayor de cuadros negativos con el reemplazo hormonal. Las mujeres sometidas a TRH diagnosticadas con cáncer de mama en etapa anterior, especialmente del subtipo que expresa receptores de estrógeno, muestran incremento en las cifras de recidiva.

Uso de ACO: en la incidencia de este tipo de cáncer también intervienen el consumo de anticonceptivos VO, estos elevan de forma moderada el riesgo de cáncer mamario y también el notable efecto protector contra los cánceres del epitelio ovárico y de endometrio (9). Un 
metaanálisis reciente que evalúa el peso de los diferentes factores de riesgo en mujeres de 40 a 49 años evidencia un posible incremento del riesgo de 1-1,5\%. Parece que el riesgo podría ser mayor si se administran en etapas finales de la vida reproductiva, cuando la mujer es más susceptible al desarrollo de esta enfermedad.

\section{CLASIFICACIÓN}

En general, se dividen en carcinoma in situ y en enfermedad invasiva, y estos a su vez, pueden ser ductales ó lobulares. El Carcinoma ducal infiltrante, que proviene del epitelio ductal, es el tipo más común y constituye el $70-80 \%$ de los casos. El lobular infiltrante abarca un $8 \%$ y hay subtipos mixtos con afectación ductal y globular que constituyen un $7 \%$ de los diagnósticos. Por otro lado, hay también subtipos menos comunes como el carcinoma metaplásico, mucinoso, tubular, medular y papilar, que todos juntos forman menos del $5 \%$ de las neoplasias malignas de seno (6).

En la clasificación in situ nos encontramos el carcinoma ductal in situ (DCIS) y el carcinoma lobulillar in situ (LCIS). EI LCIS es una lesión que ocurre en los ductos lobulares terminales y que ha sido removida de la patología cancerígena por el American Joint Committee on Cancer (14). Por otro lado, el DCIS es un grupo heterogéneo que se caracteriza por el tamaño de la lesión, grado nuclear, presencia y extensión de necrosis en comedón y patrón arquitectónico (3). Es menos común en mujeres menores de 30 años, sin embargo, aumenta con la edad, detectándose en 0.6 por 1000 tamizajes en mujeres de 40 a 49 años (14).

\section{CLÍNICA}

El cáncer de mama en etapas iniciales se presenta de manera subclínica en la mayoría de los casos, es decir que solamente es detectable por estudios de imagen, en menor proporción por clínica; no es posible distinguir entre una lesión benigna y una maligna, ni un quiste de una masa mamaria sólida en la exploración clínica inicial. Sin embargo, los hallazgos de la exploración física, interpretados junto con las imágenes y resultados de patología contribuyen mucho a las decisiones terapéuticas.

Exploración física: comienza con una adecuada postura del paciente, en posición vertical, sentada inicialmente y luego acostada con las manos detrás de la cabeza. Se inicia con la inspección en búsqueda de diferencias en el tamaño, cambios cutáneos, retracción del pezón, patrones venosos prominentes y signos inflamatorios. Se registra la presencia y características de la secreción del pezón (13). La localización de una masa se describe de forma específica según su posición de acuerdo a las manecillas del reloj y luego se mide sobre el eje longitudinal con una regla. Se especifica la distancia del centro del pezón al centro de la tumoración (7). El dato más útil en el examen físico es la localización y el tamaño de la tumoración. Aunque la exploración clínica únicamente nunca permite descartar la malignidad, existen rasgos benignos como superficie lisa, redondez y movilidad. También se debe explorar de forma cuidadosa y detallada las axilas, fosa infraclavicular y fosa supraclavicular para identificar linfadenopatía (13). 


\section{DIAGNÓSTICO Y TAMIZAJE}

Mamografía: una gran parte de la disminución de la mortalidad se ha asociado a la detección en estadios tempranos mediante mamografía, en la cual se reporta que en un 25 a $30 \%$ corresponde a carcinoma ductal in situ (4). Se ha visto que puede detectar carcinomas incluso 1.5 años a 4 años antes de ser clínicamente evidente (15). Hallazgos anormales en el estudio detectan gran parte de los carcinomas mamarios. Sin embargo debe de complementarse con otros métodos para confirmar el diagnóstico, ya que algunos de los cánceres más agresivos aparecen normales en la mamografía (4).

La incidencia de cáncer de mama es proporcional al aumento de la edad y factores de riesgo asociados, por lo que, debido a la incidencia en pacientes de menos de 40 años con riesgo promedio de padecerlo, no es recomendado realizar el tamizaje. También se ha documentado mayor cantidad de falsos positivos en estas pacientes (15). Algunas mujeres jóvenes pueden presentar masas mamarias de gran tamaño antes de la edad de tamizaje, en estos casos sí es recomendable realizar mamografía entre los estudios diagnósticos iniciales (4).

Se ha establecido que la toma de decisión para inicio de tamizaje en mujeres de 40 años, debe de ser compartida entre médico y paciente, tomando en cuenta el riesgo-beneficio de la realización. Las mujeres que sí opten por iniciar el tamizaje a los 40 años, y cuyos hallazgos en el mismo sean normales, se les recomienda seguimiento cada 2 años (15).

El Breast Cancer Surveillance Consortium, fundado por el National
Cancer Institute, documenta que de más de 1.8 millones de mamogramas convencionales realizados, entre los años 2004 y 2008, en mujeres de entre 18 y >80 años, obtuvieron una sensibilidad de $84.4 \%$ y una especificidad de $90.8 \%$. Siendo $9.6 \%$ el índice de repetición y $4.3 \%$ el valor predictivo positivo. Asimismo, en el "Twenty five year followup for breast cancer incidence and mortality of the Canadian National Breast Screening Study: randomised screening trial", nueve estudios randomizados controlados durante 25 años, con más de 650,000 mujeres tamizadas, reportaron que hay un efecto protector en las pacientes de entre 40 a 69 años tamizadas con mamografía, versus las no tamizadas (16).

Aún así, aunque la información parezca favorable, estudios a más largo plazo reportan que el beneficio absoluto versus las muertes prevenidas es relativamente bajo. Evidenciado en el metaanálisis "Effectiveness of Breast Cancer Screening: Systematic Review and Metaanalysis to Uptodate the 2009 U.S Preventive Services Task Force Recommendation", nueve estudios randomizados, donde fueron tamizadas 10,000 mujeres durante 10 años, y solo 3 muertes pudieron ser prevenidas (15).

En cuanto la diferencia entre la mamografía convencional y la digital, el Digital Mammographic Imaging Screening Trial (DMIST) reportó que la precisión de ambos era similar, no obstante, en mujeres de menos de 50 años, premenopáusicas, perimenopáusicas y con mamas densas, había mayor precisión en la mamografía digital, pero también se asociaba a un aumento de falsos positivos (16). 
El American College of Radiology, creó la clasificación Breast Report Imaging and Data System (BI-RADS), para establecer una relación entre los hallazgos mamográficos y su respectiva recomendación. Las pacientes con síntomas de mama específicos, como nódulo palpable, secreción por pezón, dolor focalizado, deben realizarse una mamografía diagnóstica. En el BI-RADS la categoría 0 indica que son necesarios más estudios complementarios, ya sea ultrasonido o raramente Resonancia magnética. Las categorías 4c y 5 alertan sobre alta sospecha de malignidad (17). Ver TABLA 1.

La presencia de masas o densidades de tejido blando con formas espiculadas y calcificaciones agrupadas son indicativos de cáncer de mama. (3). Resultados negativos en la mamografía pero con sospecha clínica se deben estudiar más a fondo debido a la tasa de falsos negativos del estudio (10-30\%), siendo mayor en mujeres de mamas densas (17).

Ultrasonido: el rol principal del ultrasonido es el seguimiento de mamas anormales en la mamografía. Evalúa las masas o asimetrías y diferencia lesiones quísticas de sólidas, además de ser guía para la realización de biopsias y marcajes para quimioterapia neoadyuvante $(4,16,17)$.

Es apropiada para la evaluación de implantes mamarios en mujeres con contraindicación para RM o donde ésta no esté disponible. Mediante el ultrasonido se puede inspeccionar la integridad de la cápsula de silicón en pacientes con implantes mamarios, así como derrames del mismo en tejido circundante, es posible observar una imagen en "tormenta de nieve" o cuando hay ruptura intracapsular se observa imagen en "escalera". Asimismo puede utilizarse para la evaluación de nódulos linfáticos axiliares que sean sospechosos de metástasis (17).

\begin{tabular}{|c|c|c|}
\hline Categoría & Manejo & $\begin{array}{l}\text { Probabilida } \\
\text { d de cáncer }\end{array}$ \\
\hline $\begin{array}{l}\mathbf{0 :} \\
\text { Incompleto }\end{array}$ & $\begin{array}{l}\text { Necesidad de } \\
\text { imágenes } \\
\text { adicionales. }\end{array}$ & $\mathrm{N} / \mathrm{A}$ \\
\hline 1: Negativo & $\begin{array}{c}\text { Mamografía de } \\
\text { rutina }\end{array}$ & $0 \%$ \\
\hline 2: Benigno & $\begin{array}{c}\text { Mamografía de } \\
\text { rutina }\end{array}$ & $0 \%$ \\
\hline $\begin{array}{l}\text { 3: } \\
\text { Probableme } \\
\text { nte benigno }\end{array}$ & $\begin{array}{c}\text { Seguimiento a } 6 \\
\text { meses o vigilancia } \\
\text { continua mediante } \\
\text { mamografía. }\end{array}$ & $\begin{array}{l}>0 \% \text { pero } \\
\text { menor/igual } \\
\quad \text { a } 2 \%\end{array}$ \\
\hline $\begin{array}{l}\text { 4: } \\
\text { Sospechoso } \\
\text { de } \\
\text { malignidad }\end{array}$ & Biopsia tisular & $\begin{array}{l}>2 \% \text { pero } \\
\text { menor a } \\
95 \%\end{array}$ \\
\hline $\begin{array}{r}\text { 4a: Baja } \\
\text { sospecha }\end{array}$ & & $\begin{array}{c}>2 \% \text { a } \\
\text { menor/igual } \\
\text { a } 10 \%\end{array}$ \\
\hline $\begin{array}{l}\text { 4b: } \\
\text { Moderada } \\
\text { sospecha }\end{array}$ & & $\begin{array}{l}>10 \% \text { a } \\
\text { menor/igual } \\
\text { a } 50 \%\end{array}$ \\
\hline $\begin{array}{c}\text { 4c: Alta } \\
\text { sospecha }\end{array}$ & & $\begin{array}{l}>50 \% \text { a } \\
\text { menor a } \\
95 \%\end{array}$ \\
\hline $\begin{array}{l}\text { 5: Muy } \\
\text { sugestivo de } \\
\text { malignidad }\end{array}$ & Biopsia tisular & $\begin{array}{c}\text { Mayor/igual } \\
95 \%\end{array}$ \\
\hline $\begin{array}{l}\text { 6: } \\
\text { Malignidad } \\
\text { confirmada } \\
\text { en biopsia }\end{array}$ & $\begin{array}{l}\text { Excisión } \\
\text { quirúrgica }\end{array}$ & $\mathrm{N} / \mathrm{A}$ \\
\hline \multicolumn{3}{|c|}{$\begin{array}{l}\text { Fuente: Sickles EA, D'Orsi CJ, Bassett LW, et al. ACR } \\
\text { BI-RADS } \AA \text { Mammography. In: ACR BI-RADS® Atlas } \\
\text { Breast Imaging Reporting and Data System. Reston, VA } \\
\text { American College of Radiology; 2013. (20) }\end{array}$} \\
\hline
\end{tabular}

\section{8}


Presencia de espiculaciones, hipoecogenicidad, microlobulación, calcificaciones internas, sombreado, lesiones más altas que anchas, y márgenes angulados, son hallazgos de malignidad en el ultrasonido (3).

Resonancia Magnética: pese no ser un estudio de rutina, es mucho más sensible que la mamografía, el ultrasonido o el examen físico. Identifica enfermedad adicional (ipsilateral) en un $16 \%$ de mujeres con cáncer de mama, siendo el sitio más frecuente de encontrar tumor en una mama post mastectomizada. Además es más precisa que los estudios convencionales de imágenes para el estimado de la extensión de las lesiones, mejorando así el abordaje quirúrgico. De igual forma mejora la detección en mamas muy densas y en pacientes con cáncer oculto en la mamografía. Aun así, su uso para la detección de lesiones contralaterales es controversial, se recomienda en casos de mujeres con alto riesgo de enfermedad contralateral, y en pacientes las cuales serán sometidas a reconstrucción de la mama con reducción o mastectomía contralateral (4).

Su función está basada en la angiogénesis y neovascularidad de los tumores malignos. Lo vasos asociados a estos tienen permeabilidad aumentada, lo que hace que el medio de contraste utilizado (Gadolinio intravenoso) se libere rápidamente y así logra distinguir malignidad de crecimientos benignos. En pacientes con implantes de silicón que requieran inspección, no es necesario el uso de contraste $(4,18)$.

Su uso debe ser reservado para dilemas diagnósticos o tomas de biopsias, debido a la tasa significativa de falsos positivos. Está contraindicado en embarazo, dispositivos implantables y cuerpos extraños, función renal disminuida, y alergia al Gadolinio $(18,19)$.

Biopsia de mama: En pacientes con masa mamaria palpable o mamografía sospechosa, la biopsia percutánea es mandatoria y de elección sobre la biopsia quirúrgica. En general, la biopsia con aguja gruesa, ofrece un diagnóstico histológico más definitivo por favorecer la toma de una muestra más amplia y evitar la toma de muestras inadecuadas. Permite además distinguir entre carcinoma in situ y carcinoma invasivo (20).

Biopsia del ganglio centinela: consiste en inyectar de manera prequirúrgica un marcador radiosensible cerca del área de sospecha de la lesión o a nivel periareolar, que drena hacia el primer ganglio al que se hizo metástasis, el cual se localiza con una sonda gamma y se reseca para biopsiarlo (21).

La evaluación prequirúrgica de los nódulos linfáticos axilares, separa a los pacientes en 2 categorías: aquellos que presentan nódulos axilares clínicamente positivos, y aquellos con nódulos negativos. Pacientes con nódulos negativos, se someten a una biopsia del ganglio centinela. En cuanto a los pacientes con nódulos positivos, pacientes con una biopsia positiva del ganglio centinela, dudas en la correlación clínica y patológica, estados avanzados y carcinoma inflamatorio; se les realiza una disección axilar formal con linfadenectomía (21).

Inmunohistoquímica: Es claro que el cáncer de mama depende de estrógenos y progesterona para su crecimiento, y que esos efectos son mediados a través de receptores hormonales (22). 
Si se diagnostica un carcinoma, se debe realizar un adecuado estiaje local y distal de la enfermedad. (11) Los expertos recomiendan determinar también el grado histológico, la expresión de receptores de estrógeno, de progesterona, el Ki67 y la sobreexpresión de Her-2. Esto es crítico para fines diagnósticos, evaluar el pronóstico y establecer las opciones terapéuticas tales como hormonoterapia, quimioterapia y terapia anti Her-2 (23).

El análisis de biomarcadores en el cáncer de mama inició con la determinación de receptores hormonales para el uso de tratamiento con tamoxifeno (23).

No todos los pacientes con cáncer de mama se benefician de terapia endocrina, sino que los que mejor responden son los que tienen receptores de estrógenos y progesterona positivos. Tumores que no expresan estos receptores, no responden adecuadamente a terapia hormonal y pueden beneficiarse de quimioterapia citotóxica (22).

La sobreexpresión de Her-2, predice un beneficio de la terapia dirigida hacia estos receptores (6). Según diversas investigaciones, aproximadamente $80 \%$ de los cánceres de mama expresan receptores de estrógenos y progesterona, $23 \%$ sobreexpresa el Her-2 y $13 \%$ no expresaba ni receptores hormonales ni Her-2, por lo que molecularmente se expresaban como triple negativos (6).

EI KI67, es un marcador protéico utilizado para evaluar la actividad proliferativa del cáncer de mama. Algunas guías consideran como actividad proliferativa baja, niveles de Ki-67 por debajo del 10\%, y alta mayor al $30 \%$ y de acuerdo a un metaanálisis reciente, un ki67 mayor a un $25 \%$ se asocia a un peor pronóstico. Sin embargo, la guía de la American Society of Clinical Oncology (ASCO), sigue sin recomendar su utilización y reporta que se necesitan más estudios colaborativos internacionales para estandarizar sus valores (23).

La principal utilidad clínica del Ki67, radica en la diferenciación de los subtipos Luminal A y Luminal B cuando hay receptores de estrógenos positivos pero Her-2 negativos, y así guiar el uso de terapia adyuvante o neoadyuvante (24).

Clasificación molecular: la clasificación molecular, realizada mediante una inmunohistoquímica a la muestra biopsiada, proporciona información acerca de la probabilidad de recidiva y la ventaja o no de recibir quimioterapia adyuvante. Molecularmente, estos tumores se clasifican en luminal-A, luminal-B, tumores que sobreexpresan el Her-2 y los Basal-like ó triple negativos (24).

El subtipo luminal-A representa más del $50 \%$ de las neoplasias, suele ser de un bajo grado hlitológico y presentan el pronóstico más favorable con una supervivencia a 5 años superior al 80\%; en estos tipos, es frecuente la metástasis a hueso (25). Por lo general, presentan baja proliferación, alta respuesta al tratamiento hormonal y buen pronóstico (24).

El luminal-B tiene una incidencia del $15 \%$, con una tasa de supervivencia a 5 años de $40 \%$ (24). Este subtipo, tiende a mostrar una mayor proliferación, menor respuesta endocrina y un pronóstico más pobre (24).

Tumores que sobreexpresan Her-2, tienen una incidencia de $15-30 \%$, un grado histológico medio 0 alto con tendencia a la multifocalidad, con sobrevida a 5 años del $31 \%$ antes de la 
introducción del trastuzumab. Son frecuentes las metástasis a hueso, cerebro, hígado y pulmón. Responden a Trastuzumab, Pertuzumab y Lapatinib (23).

Las neoplasias basales ó triple negativas, constituyen apenas un $12-17 \%$ de los casos y conllevan una alta mortalidad. Presenta mayor incidencia en mujeres jóvenes, afroamericanas y en portadoras de una mutación en el gen BRCA-1. Tienden a ser unifocales, con un alto grado histológico y producen metástasis axilares frecuentes y recidivas, especialmente durante los primeros 4 años, sin embargo, después de los 10 años de control, es menor que en pacientes con receptores hormonales positivos.

Las malignidades triple negativas presentan mayor sensibilidad a la quimioterapia neoadyuvante (25). Ver TABLA 2.

\section{TRATAMIENTO}

\section{Enfermedad temprana}

- Cirugía: inicialmente en estos casos el manejo es quirúrgico. La cirugía incluye la resección del tumor primario con márgenes negativos, ya sea mediante cirugía conservadora o mastectomía (26). Siempre se debe realizar exploración de la axila con búsqueda del ganglio centinela o con disección radical. De acuerdo con la modalidad de tratamiento quirúrgico primario seleccionada y el reporte histopatológico definitivo, se determina la necesidad de ofrecer adyuvancia sistémica o local con radioterapia (12).

\begin{tabular}{|c|c|}
\hline \multicolumn{2}{|c|}{$\begin{array}{l}\text { TABLA 2. Clasificación } \\
\text { cáncer de mama } \\
\text { inmunohistoquímica }\end{array}$} \\
\hline $\begin{array}{l}\text { Subtipo } \\
\text { molecular }\end{array}$ & Expresión \\
\hline Luminal-A & $\begin{array}{l}\text { RE y RP positivos , Her-2 } \\
\text { negativo, Ki67 bajo }\end{array}$ \\
\hline Luminal-B & $\begin{array}{l}\text { RE y RP positivos, Her-2 } \\
\text { positivo, Ki67 intermedio }\end{array}$ \\
\hline $\begin{array}{l}\text { Sobreexpresión } \\
\text { de Her-2 }\end{array}$ & $\begin{array}{l}\text { RE y RP negativos, Her-2 } \\
\text { positivo, Ki67 alto }\end{array}$ \\
\hline Triple negativo & $\begin{array}{l}\text { RE y RP negativos, Her-2 } \\
\text { negativo, Ki67 alto }\end{array}$ \\
\hline \multicolumn{2}{|c|}{$\begin{array}{l}\text { RE: receptores de estrógenos, RP: receptores de } \\
\text { progesterona, Her-2: receptor } 2 \text { de factor de crecimiento } \\
\text { epidérmico humano. }\end{array}$} \\
\hline \multicolumn{2}{|c|}{$\begin{array}{l}\text { Fuente: Merino J, Ros L, Tabanera M, et al. El cáncer de } \\
\text { mama en el siglo XX1: de la detección precoz a los nuevos } \\
\text { tratamientos. Radiología. } 2017 \text {. (25) }\end{array}$} \\
\hline
\end{tabular}

Hay que recordar que existen casos en los que se debe realizar mastectomía como primera opción; cuando existe contraindicación para recibir radioterapia, enfermedad multicéntrica, dificultad para obtener márgenes adecuados y cuando se desea un resultado cosmético favorable. En varios ensayos clínicos con seguimiento a 30 años, se ha podido demostrar que la cirugía conservadora de mama combinada con radioterapia ofrece los mismos porcentajes de supervivencia global que la mastectomía (26).

- Manejo de la axila: a lo largo de tiempo la disección de la axila ha sido un procedimiento estándar. A pesar de esto, el beneficio de este procedimiento cuando existen ganglios negativos desde la perspectiva clínica no ha sido demostrada, por lo que en este grupo de pacientes se ha adoptado la técnica del ganglio centinela. $(12,26)$

El estudio NSABP B-32 y un 
metaanálisis han confirmado la eficacia de esta técnica. La combinación de ambas permite la identificación del GC en más de $96 \%$ de los casos, con $<5 \%$ de falsos negativos (26).

\section{Enfermedad localmente avanzada}

- Manejo de la axila: en pacientes con T3 y axila negativa que reciben tratamiento neoadyuvante, existe controversia con respecto al momento óptimo para ofrecer ganglio centinela (26). Las guías de la Red Nacional Integral de Cáncer (NCCN) recomiendan la implementación del ganglio centinela previo la quimioterapia neoadyuvante porque ofrece mayor información pronóstica para la planeación del tratamiento locorregional. La disección de región axilar complementaria se realiza al momento de la operación definitiva cuando el GC es positivo. La implementación de ganglio centinela de repetición en el escenario de posneoadyuvancia en pacientes con GC inicialmente positivo está contraindicada gracias a las altas tasas de falsos negativos (26).

- Quimioterapia adyuvante: el principal objetivo de esta terapia es eliminar la enfermedad micrometastásica, ya q la recurrencia sistémica es la principal causa de muerte en estas pacientes (12). La indicación del tratamiento sistémico se basa en factores del tumor (tamaño, grado de diferenciación, estado ganglionar, receptores hormonales, sobreexpresión o amplificación de HER-2, Ki-67, entre otros) y factores relacionados con la paciente (edad, comorbilidades, estado funcional) (8).

- Trastuzumab adyuvante: es un anticuerpo monoclonal humanizado dirigido contra la proteína HER-2. Diversos estudios retrospectivos han demostrado que los tumores menores de $1 \mathrm{~cm} \mathrm{HER}-2$ positivos tienen un riesgo incrementado de recurrencia y muerte, también se ha visto el beneficio de un año de tratamiento con trastuzumab en recurrencia $y$ muerte en los tumores menores de 2 $\mathrm{cm}$ con ganglios negativos; por ello, las guías del NCCN sugieren el empleo de trastuzumab y quimioterapia en mujeres con tumores T1b HER-2 positivo $(8,12)$. Se conoce que este medicamento aumenta el riesgo de toxicidad cardíaca, que se manifiesta como disminución asintomática de la fracción de eyección y con menor frecuencia por insuficiencia cardiaca clínica (26). Se recomienda realizar ecocardiograma cada tres meses en pacientes con factores de riesgo (obesidad, hipertensión, FEVI limítrofe) o cada cuatro a seis meses en pacientes sin factores de riesgo. El beneficio de otros tratamientos biológicos como el del lapatinib o bevacizumab en el contexto adyuvante no ha sido demostrado (26).

- Terapia endocrina adyuvante con receptores hormonales positivos: el tamoxifeno es un miembro de la familia de los moduladores selectivos de los receptores estrogénicos (SERM) (12). Cinco años de tamoxifeno han demostrado una disminución en el riesgo de 
recurrencia de $40 \%$ y en el riesgo de muerte de $35 \%$, lo que se traduce en un beneficio absoluto del riesgo de recaída a 15 años de $12 \%$ y del riesgo de muerte de $9 \%$ (26). La dosis de 20 mg al día es la óptima, sin que exista beneficio en dosis mayores. Los beneficios de este son similares en mujeres premenopáusicas que en posmenopáusicas. Sin embargo, es importante tener en cuenta que el uso de este fármaco implica un riesgo de desarrollar cáncer endometrial de 1\% y de enfermedad tromboembólica de $2 \%$, así como ganancia de peso, depresión, disfunción sexual, síntomas vasomotores y síntomas ginecológicos (12).

- Ablación ovárica: en mujeres premenopáusicas con cáncer de mama con receptores hormonales positivos, esta terapia ha demostrado una disminución de la recurrencia y la mortalidad. Los beneficios son similares a los que se obtienen con la quimioterapia. La ablación ovárica puede conseguirse con cirugía, radiación o agonistas de la hormona liberadora degonadotropinas $(\mathrm{GnRH})$ (26).

- Radioterapia adyuvante: está indicado en todos los pacientes que hayan sido sometidos a cirugía conservadora. Dos estudios aleatorizados demostraron reducción de las recurrencias locales. El riesgo de recurrencia posmastectomía se relaciona con el número de ganglios axilares positivos. La radioterapia se usa en las pacientes con cuatro o más ganglios metastásicos, evidencia de extensión extracapsular, márgenes quirúrgicos cercanos, tumor mayor de
$5 \mathrm{~cm}$ de diámetro e invasión de la piel (12).

\section{Enfermedad metastásica}

La enfermedad metastásica puede clasificarse como de alto y bajo riesgo. Se define como de bajo riesgo aquellos pacientes con receptores hormonales positivos, HER-2 negativo, periodo libre de enfermedad mayor de dos años, y enfermedad metastásica limitada a tejidos blandos y hueso, con una supervivencia global de 3 a 5 años (12). En estos casos, el tratamiento de primera elección es el hormonal. Por alto riesgo se entienden los casos con receptores hormonales negativos, HER-2 positivo, intervalo libre de enfermedad mayor de dos años, enfermedad metastásica extensa, y afección visceral con una supervivencia aproximada de 12 meses (8). El tratamiento de elección es con quimioterapia $\mathrm{y} / \mathrm{o}$ trastuzumab $\mathrm{u}$ otras terapias biológicas.

\section{Enfermedad recurrente}

- Recaída local posmastectomía: por lo general, la mayoría de las recaídas ocurre en la cicatriz dejada por la resección del tumor primario o cerca de ésta y se presenta como uno o más nódulos cutáneos o subcutáneos asintomáticos. Algunas pacientes se presentan con invasión difusa de la pared torácica e induración más allá de los límites del tratamiento local, lo cual se denomina carcinoma en coraza. Un $80 \%$ de las recaídas locales ocurren dentro de los primeros 5 años desde el tratamiento. El periodo libre de enfermedad es el mejor indicador de la supervivencia global. La radioterapia es el estándar 
de tratamiento de las pacientes con recaída local (26).

- Recaída local posterior a cirugía conservadora: la mayoría ocurren a nivel de la cicatriz de la mastectomía o cercana a ella, entre los 2 a 7 años posterior al tratamiento. Parece tener mejor pronóstico la recaída después del tratamiento conservador en comparación con la recaída local después de la mastectomía. El tratamiento estándar de una recaída local aislada es la mastectomía, cuya supervivencia libre de enfermedad a 5 años es de 60 a $75 \%$ (26).

\section{CONCLUSIÓN}

El riesgo de padecer cualquier tipo de cáncer incrementa con la edad, en parte por la exposición mantenida a múltiples factores de riesgo. En el caso del cáncer de mama, éste aumenta principalmente a partir de la quinta década de vida y es la neoplasia maligna más frecuente en mujeres, sin embargo, su etiología aún es poco conocida. Se plantea que una de cada 14 mujeres podría presentar cáncer de mama en algún momento de su vida (8). A toda paciente con sospecha de cáncer de mama, debe realizársele un adecuado examen físico, pruebas de imágenes, estudios histológicos y moleculares, no solo para contar con un adecuado estadiaje local y sistémico, sino también para obtener información acerca del comportamiento, y la respuesta del tumor a diferentes terapias hormonales y citotóxicas beneficiosas para la paciente. De igual manera, la prevención y el diagnóstico temprano de la enfermedad es fundamental para la sobrevida de las afectadas y en esto radica la importancia de la realización de campañas educativas, de los sistemas de tamizaje y avances tecnológicos en la medicina moderna.

\section{REFERENCIAS}

1. Lugones M, Ramírez M. Aspectos históricos y culturales sobre el cáncer de mama. Rev Cubana Med Gen Integr [Internet]. 2009 Sep [citado 2019 Mar 12]; 25( 3 ). 160-166. Disponible en: http://scielo.sld.cu/scielo.php?script=sci arttext\&pid=S0864-21252009000300020\&lng=es

2. Ruiz S, Villanueva A. Evolución del cáncer de mama a través de la historia. Gaceta Médica de Bilbao. 2000; 97(2): 35-36. https://doi.org/10.1016/S0304-4858(00)74324-1.

3. Esserman L, Joe B. Clinical features, diagnosis, and staging of newly diagnosed breast cancer [Internet]. Waltham (MA): UpToDate Inc; 2019 [ cited 2019 March 09]. Available from: https://www.uptodate.com/contents/clinical-features-diagnosis-and-staging-of-newly-diagnosed-breastcancer?search=clinical\%20features\%20breast\%20cancer\&source=search result\&selectedTitle=1 150\&u sage type=default\&display rank=1.

4. Esserman L, Joe B. Diagnostic evaluation of women with suspected breast cancer [Internet]. Waltham (MA): UpToDate Inc; 2019 [cited 2019 March 06]. Available from: https://www.uptodate.com/contents/diagnosticevaluation-of-women-with-suspected-breast-cancer?source=mostViewed widget

5. Aguiar G, Sánchez C. Cáncer de mama en mujeres jóvenes ¿por qué está aumentando? [Internet]. Michoacán (CDMX): Fundación Cimab; 2017 [cited 2019 March 06]. Available from: http://www.cimab.org/articulos-de-interes/cancer-de-mama-en-mujeres-jovenes-por-que-esta-aumentando 
6. Troyan S, MD. Breast cancer in women. DynaMed [Internet]. Massachusetts: EBSCO Industries; 2018 [cited 2019 March 19]. Available from: https://search.ebscohost.com/login.aspx?direct=true\&db=dme\&AN=113654\&site=dynamedlive\&scope $=$ site

7. Troyan S, MD. Early and locally advanced noninflammatory breast cancer [Internet]. Massachusetts:EBSCO Industries; 2018 [cited 2019 March 19]. Available from: https://search.ebscohost.com/login. aspx?direct=true\&db=dme\&AN=921332\&site=dynamedlive\&scope $=$ site

8. Fauci AS, Hauser SL, Jameson J, Kasper DL, , Longo DL, Loscalzo J. Cáncer de mama-Harrison Principios de Medicina Interna [Internet]. New York (NY):McGraw-Hill Global Education Holdings LLC; 2018 [Recuperado el 15 de marzo de 2019]. Disponible en: https://accessmedicina.mhmedical.com/content. aspx?sectionid=203644540\&bookid=2461\&Resultclick=2\# $\underline{1161977997}$

9. Vich P, Brusint B, Álvarez-Hernández C, Cuadrado-Rouco C, Diaz-García N, Redondo-Margüello E. Actualización del cáncer de mama en Atención Primaria (I/V). SEMERGEN - Medicina de Familia. 201409 ;40(6): 326-333. https://doi.org/10.1016/..semerg.2014.02.012

10. Vial MT, Ibarra Á. Anatomía patológica y tumores hereditarios. Revista Médica Clínica Las Condes. 2017 07; 28(4): 591-597. https://doi.org/10.1016/..rmclc.2017.07.002

11. Bland K, Hunt KK, Robertson JR, I. (2015). Mama - Schwartz principios de cirugía [Internet]. New York (NY):McGraw-Hill Global Education Holdings LLC; 2015 [Recuperado el 25 de marzo de 2019]. Disponible en:

https://accessmedicina.mhmedical.com/content.aspx?bookid=1513\&sectionid=98625451\&jumpsectionid= 98625572\#1119716502

12. Bradshaw KD, Corton MM, Halvorson LM, Hoffman BL, Schorge JO, Schaffer JI. Enfermedad mamaria Williams Ginecología [Internet]. New York (NY): McGraw-Hill Global Education Holdings LLC; 2017 [Recuperado el 20 de marzo de 2019]. Disponible en: https://accessmedicina.mhmedical.com/content.aspx?bookid=2162\&sectionid=165576510\&jumpsectionid $=165576620 \# 1144481688$

13. Esserman L, Joe B. Clinical features, diagnosis, and staging of newly diagnosed breast cancer [Internet]. Waltham (MA): UpToDate Inc; 2019 [cited 2019 March 09]. Available from: https://www.uptodate.com/contents/clinical-features-diagnosis-and-staging-of-newly-diagnosed-breastcancer?search=clinical\%20features\%20breast\%20cancer\&source=search result\&selectedTitle=1 150\&u sage type=default\&display rank $=1$

14. Collins L, Laronga C, Wong J. Breast ductal carcinoma in situ: Epidemiology, clinical manifestations, and diagnosis [Internet]. Waltham (MA): UpToDate Inc; 2019 [cited 2019 March 11]. Available from: https://www.uptodate.com/contents/breast-ductal-carcinoma-in-situ-epidemiology-clinical-manifestations-

and-

diagnosis?search=BREAST\%20DUCTAL\&source=search result\&selectedTitle=3 76\&usage type=defaul t\&display rank=3

15. Slanetz $\mathrm{P}$, Venkataraman $\mathrm{S}$. Breast imaging for cancer screening: Mammography and ultrasonography [Internet]. Waltham (MA): UpToDate Inc; 2019 [cited 2019 March 06]. Available from: https://www.uptodate.com/contents/breast-imaging-for-cancer-screening-mammography-andultrasonography?source=autocomplete\&index=3 4\&search=BREAST\%20IMA

16. Elmore J. Screening for breast cancer: Strategies and recommendations [Internet]. Waltham (MA): UpToDate Inc; 2019 [cited 2019 March 09]. Available from: https://www.uptodate.com/contents/screeningfor-breast-cancer-strategies-and- 
recommendations? search=screening $\% 20$ breast $\% 20$ cancer \&source=search result\&selectedTitle=1 106\& usage type=default\&display rank=1

17. Elmore J. Screening for breast cancer: Evidence for effectiveness and harms [Internet]. Waltham (MA): UpToDate Inc; 2019 [cited 2019 March 08]. Available from: https://www.uptodate.com/contents/screeningfor-breast-cancer-evidence-for-effectiveness-and-harms?source=mostViewed widget

18. Slanetz P. MRI of the breast and emerging technologies [Internet]. Waltham (MA): UpToDate Inc; 2019 [cited 2019 March 09]. Available from: https://www.uptodate.com/contents/mri-of-the-breast-and-emergingtechnologies?source=mostViewed widget

19. Sabel M. Clinical manifestations and diagnosis of a palpable breast mass [Internet]. Waltham (MA): UpToDate Inc; 2019 [cited 2019 March 08]. Available from: https://www.uptodate.com/contents/clinicalmanifestations-and-diagnosis-of-a-palpable-breastmass?search=clinical\%20manifestations\%20and\%20diagnosis\%20breast\%20mass\&source=search resul $\underline{\text { t\&selectedTitle }=1 \sim 133 \& \text { usage type }=\text { default\&display } \text { rank }=1}$

20. Sickles E, D’Orsi C, Bassett L, et al. ACR BI-RADS® Mammography [Internet]. Reston (VA): American College of Radiology; 2013 [cited 2019 March 09]. Available from: https://www.acr.org/ClinicalResources/Reporting-and-Data-Systems/Bi-Rads.

21. Harlow S, Weaner D. Overview of Sentinel Lymph Node biopsy in breast cancer. [Internet]. Waltham (MA): UpToDate Inc; 2019 [cited 2018 March 05]. Available from: https://www.uptodate.com/contents/overviewof-sentinel-lymph-node-biopsy-in-breastcancer?search=Overview\%20of\%20sentinel\%20lymph\%20node\%20biopsy\%20in\%20breast\%20cancer\& source=search result\&selectedTitle=1 150\&usage type=default\&display rank=1

22. Hammond M. Hormone Receptors in breast cancer: Clinical utility and guideline recommendations to improve test accuracy [Internet]. Waltham (MA): UpToDate Inc; 2019 [cited 2018 March 05]. Available from: https:/www.uptodate.com/contents/hormone-receptors-in-breast-cancer-clinical-utility-and-guidelinerecommendations-to-improve-test-accuracy

23. Palacios Calvo J, Albanell J, Rojo F, Ciruelos E, Aranda-López I, Cortés J, García-Caballero T, Martín M, López-García MÁ, Colomer R. Consenso de la Sociedad Española de Anatomía Patológica y la Sociedad Española de Oncología Médica sobre biomarcadores en cáncer de mama. Revista Española de Patología. 2018 04; 51(2): 97-109. https://doi.org/10.1016/i.patol.2017.12.002

24. Penault-Llorca F, Radosevic-Robin N. Ki67 assessment in breast cancer: an update. Pathology. 2017 02;49(2):166-171. https://doi.org/10.1016/i.pathol.2016.11.006

25. Merino Bonilla J, Torres Tabanera M, Ros Mendoza L. El cáncer de mama en el siglo XXI: de la detección precoz a los nuevos tratamientos. Radiología. $2017 \quad 09 ; \quad 59(5)$ 368-379. https://doi.org/10.1016/i.rx.2017.06.003

26. 26. Díaz D, Dulin R, Gómez Á, Medina F, Miranda A, Navarro Y, Salinas C, Sánchez V, Sierra J. Cáncer de mama - Manual de Oncología [Internet]. New York (NY): McGraw-Hill Global Education Holdings LLC; 2017 [Recuperado el 22 de marzo de 2019]. Disponible en: https://accessmedicina.mhmedical.com/content.aspx? sectionid=180364437\&bookid=2306\&Resultclick=2\# 1150226695 